\title{
MOLECULAR DOCKING OF COMPOUNDS FROM Chaetomium Sp. AGAINST HUMAN ESTROGEN RECEPTOR ALPHA IN SEARCHING ANTI BREAST CANCER
}

\author{
Maywan Hariono $^{1 *}$, Rollando ${ }^{2}$ \\ ${ }^{1}$ Faculty of Pharmacy, Sanata Dharma University, Maguwoharjo, Sleman, Yogyakarta 55282, Indonesia \\ ${ }^{2}$ Program of Pharmacy, Faculty of Science and Technology, Ma Chung University, Malang 65151, \\ Indonesia
}

Received May 4, 2016; Accepted May 27, 2016

\begin{abstract}
A study on molecular docking-based virtual screening has been conducted to select virtual hit of compounds, reported its existence in fungal endophytes of Chaetomium sp. as cytotoxic agent of breast cancer. The ligands were docked into Human Estrogen Receptor alpha (HER $\alpha)$ as the protein which regulates the breast cancer growth via estradiol-estrogen receptor binding intervention. The results showed that two compounds bearing xanthone and two compounds bearing benzonaphtyridinedione scaffolds were selected as virtual hit ligands for HER $\alpha$ leading to the conclusion that these compounds were good to be developed as anti breast cancer.
\end{abstract}

Keywords: Molecular docking, Endophyte, Chaetomium, Breast Cancer

\section{INTRODUCTION}

In family Chaetomiaceae (Ascomyta), Chaetomium sp. is a genus employing species broadly distributed among hundred marine and terrestrial natural products (Ferlay et al, 2007; Zhang et al, 2012). To date, about 200 compounds were reported as the chemical constituents from this genus (Li et al, 2011). This genus employs either bacterial or fungal endophyte which is currently being a trending topic in searching new source of biological active compounds due to its capability to be cultivated in abundance yields (Yang et al, 2011). This is helpful to overcome the problem in isolating a single compound from raw materials which is normally using multistep extractions as well as fractionations (Hussain et al, 2012).

As characterized in general natural product compounds, these endophyte from Chaetomium sp. also posses diverse biological activities such as antibacterial, antioxidant, anti-inflammatotry, antileishmanial and many others (Ruch, Cheng, and Klaunig, 1989). The fungal endophyte is living symbiotically in between the plant cells (Strobel, 2003). Some plants have been identified its presence of Chaetomium sp. are Vinca rosea, Taxus braxifollia (Zhao et al, 2010), Phyllantus amarus
(Kandavel and Sekar, 2015), Ginko biloba (Li et al, 2011), and so forth. Uniquely, the chemicals contained in the endophyte often highly related with the identity compounds in the plant of origins. For example is vincristine, the chemotherapeutics for cancer which is contained in Vinca rosea also found in its fungal endophyte (Kumar et al, 2013). Likewise, taxol was characterized in the fungal endophyte of Taxus braxifollia (Stierle, Strobel, and Stierle, 1993).

Two mentioned plants were used clinically as the anti-cancer, a disease which is too tough to be controlled due its complicated life cycle and molecular mechanism. Breast cancer is one of the high prevalence cancers with about 1.7 million incidents annually (Lane, 2006). Therefore, the discovery and development of new anti-cancer is continuously needed especially to those resistant cancer genes.

Computational studies in a virtual identification were broadly used in the screening of natural product database as bioactive compounds. This reduces the time, budget and safety in wet lab experiment since the sample to be tested is selected based on the mathematical value of drug-receptor interaction associating with its in 
vivo pharmacodynamic behaviours (Kitchen et al, 2004). In this present study, we virtually screen 27 compounds being identified in fungal endophyte of Chaetomium sp. to be selected as the hit compounds by docking them into one of the protein targets in the breast cancer cell, i.e., human estrogen receptor alpha $($ HER $\alpha)$ DNA binding protein domain.

\section{EXPERIMENTAL}

The computer used in this study was a PC with Core 2 Duo processor $2.93 \mathrm{GHz}$ and $2 \mathrm{~GB}$ of RAM. The operating system of the PC is Windows8. Docking the compounds into human estrogen receptor alpha (HER $\alpha$; PDB 1G50) was carried out to predict affinity of chemical substances which are reported presenting in Chaetomium sp. from diverse plants. The ligands were sketched and geometrically minimized using ACD Chemsketch (www.acdlabs.com) and Marvin Sketch (www.chemaxon.com), respectively. The ligands and proteins were then prepared using AutoDockTools 1.5.6 (www.autodock.scripps.edu). The proteins were added with polar hydrogen and given by kollman charge whereas the ligands were given by gasteiger charges. The grid was centered on each protein binding site and the docking was then performed using AutoDock Vina embedded in PyRx version 8.0 (www.autodock.scripps.edu). The docking results were obeserved as free energy of binding $\left(\Delta \mathrm{G}_{\mathrm{bind}}\right)$ and the selected docking pose was visualised using Discovery Studio

\section{RESULTS AND DISCUSSION}

There have been well characterized that estrogen controls the breast cancer by stimulating the expression of certain genes (Horwitz and McGuire, 1978). Estrogen receptor with its helix $\mathrm{H} 12$ provides the binding site for estradiol as the messenger to dimerize and expose the SF-2 region. This will attract the Co-activator to bind leading to nucleic acid transcription during the breast cancer cell replication. The side chain containing ASP351 is crucial to antagonism.

The antagonist must contain an amine group of the correct basicity such that it ionizes and forms the interaction with ASP351, and it must be of the correct length and flexibility to place the amine in the correct position for binding (Patrick, 2013). Several crystal structures of human estrogen receptor $\alpha$ ligand-binding domain (hER $\alpha$ LBD) complexed with agonist or antagonist molecules have previously been solved. The binding site was characterized where in estradiol bound to, by interacting with GLU353, ARG394, PHE404 and HIS524 via electrostatics, pi-pi and hydrogen bon interaction, respectively (Eiler et al, 2001) The binding of estradiol as the chemical messenger is presented in Figure 1.

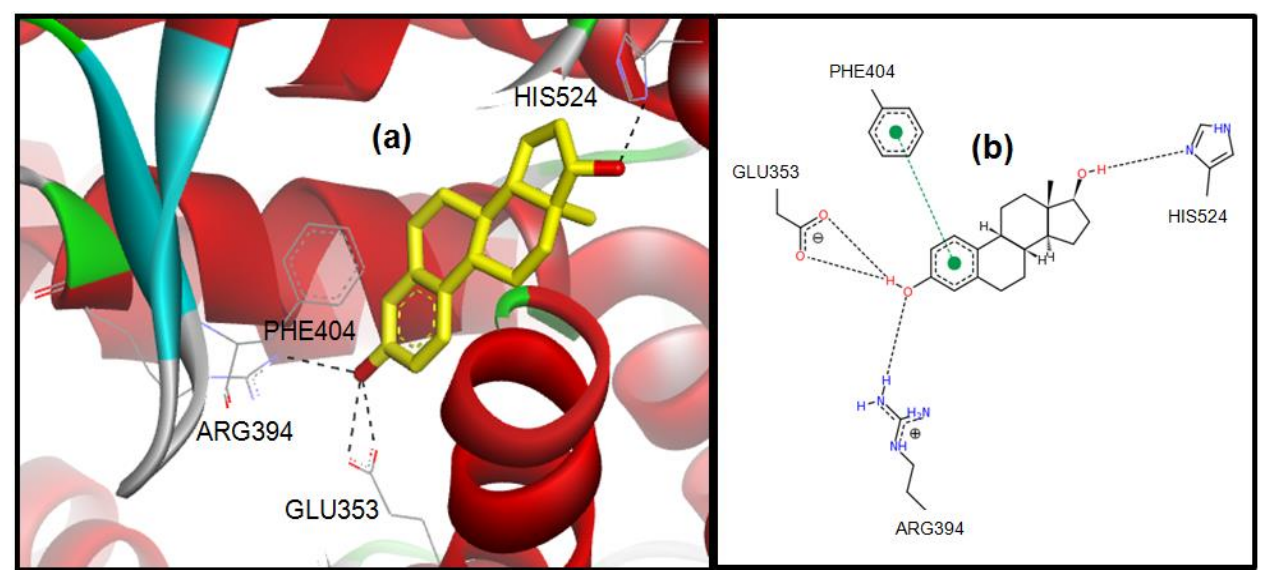

Figure 1. The chemical interaction between estradiol and HER $\alpha$ (a) 3D structure and (b) 2D structure. The protein was presented as ribbon and visualized using Discovery Studio 3.5 (www.accelrys.com) 
Jurnal Farmasi Sains dan Komunitas, 2016, 13(1), 35-43

Table 1. Compounds of Chaetomium sp. from diverse plants

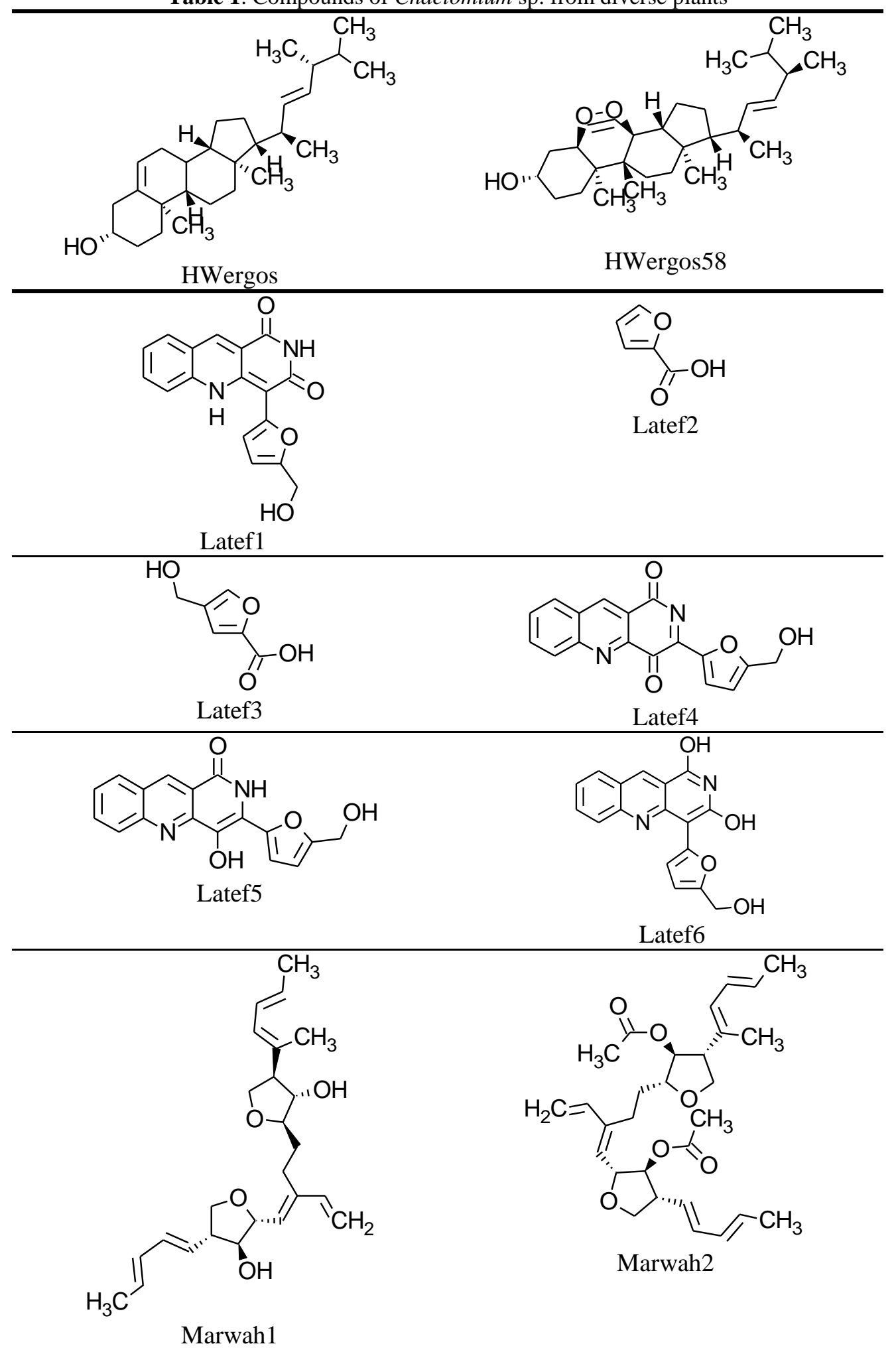



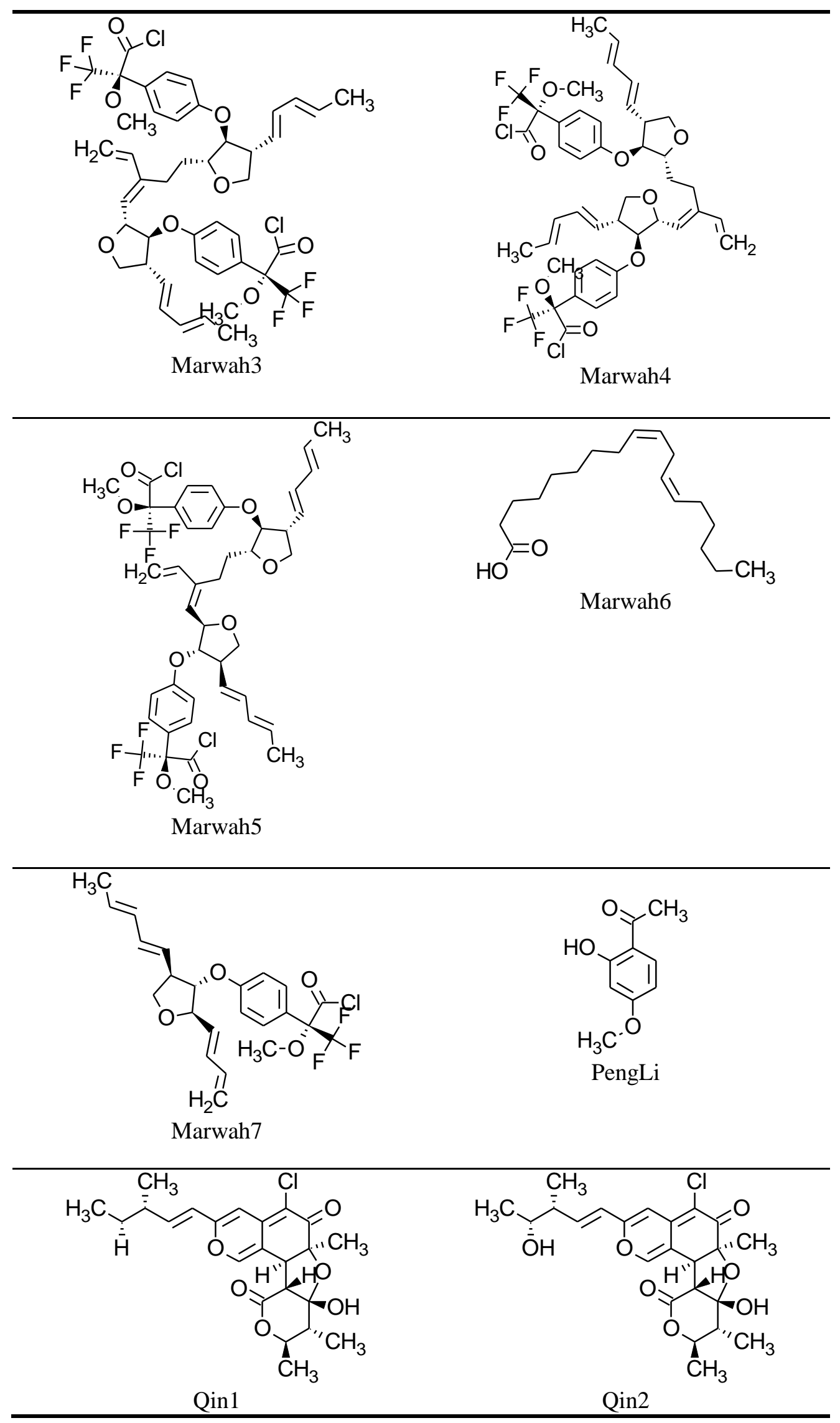


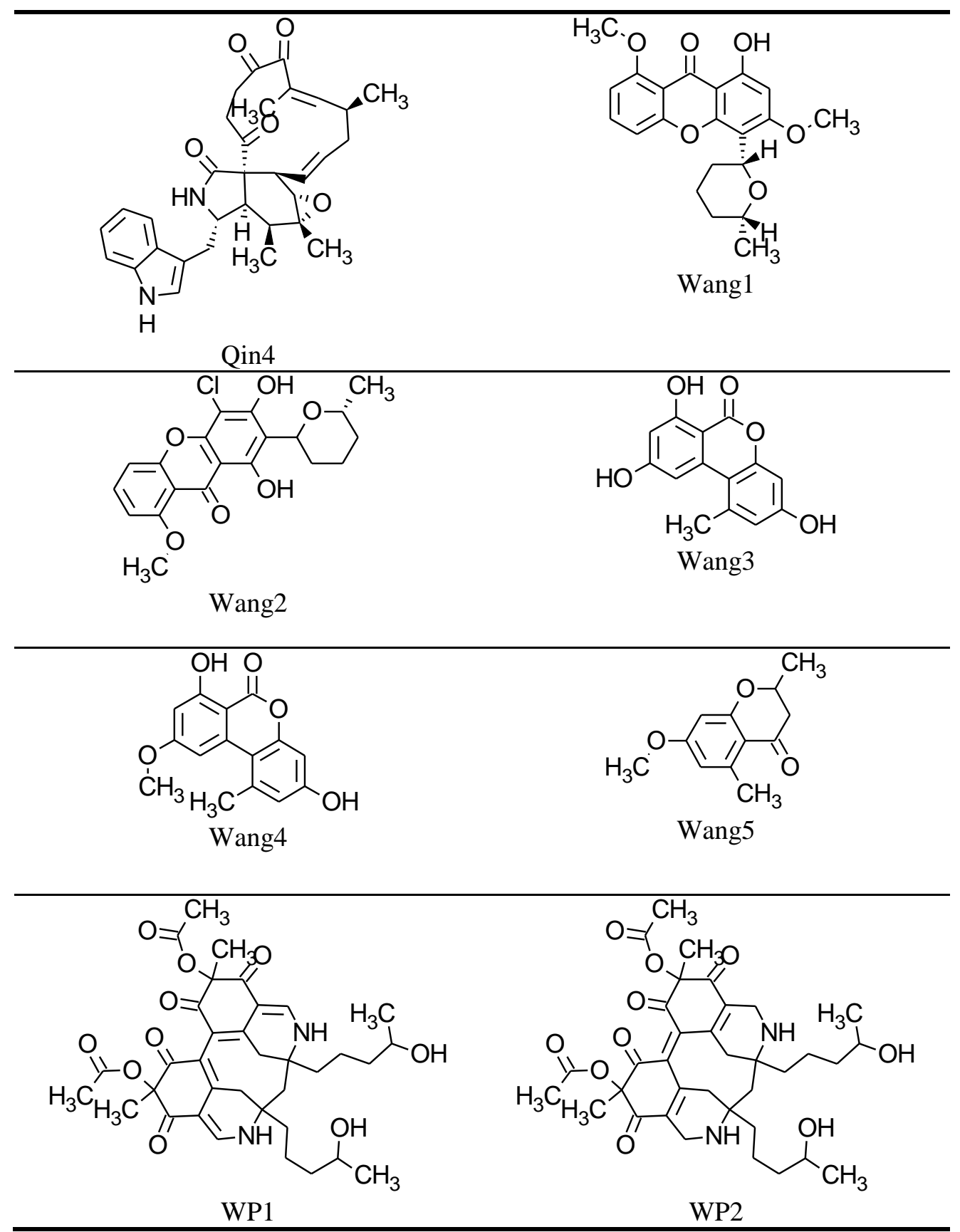

In searching new active ligands, a number of 27 compounds (see Table 1) from Chaetomium sp., was utilized as the database to be virtually screened using $1 \mathrm{G} 50$ as the protein target (Marwah et al, 2007; Abdel-Lateff, 2008; Qin et al, 2009; Li et al, 2011; Wang et al, 2012; Wang et al, 2015; Li et al, 2015;). As results, Table 2 presented the $\Delta \mathrm{G}_{\text {bind, }}$, the amino acids which contribute to its binding via $\mathrm{H}$ bonding along with its $\mathrm{H}$-bond distances. The order of compounds was ranked from the lowest $\Delta \mathrm{G}_{\text {bind }}$ to the highest one.

The $\Delta \mathrm{G}_{\text {bind }}$ was observed at range -9.2 to -4.9 $\mathrm{kcal} / \mathrm{mol}$ for virtual active compounds while inactive compounds were indicated at range -4.2 to $9.4 \mathrm{kcal} / \mathrm{mol}$. There are six compounds found to be low in $\Delta \mathrm{G}_{\text {bind }}$ but they are not bound to the active site as no hydrogen bond being observed in their poses. This might be due to the van der Waals contribution which is normally not as essential as 
hydrogen bond. Van der Waals role in the ligandprotein binding is normally to support or stabilize the pose via dipole induced dipole such as pi-pi, pication and or pi-sigma interactions. Van der waals interaction has a weak affinity, therefore, this is normally less priority in the ligand-protein interaction. Although virtual screening has been developed as a fast and accurate scoring function, however, no single scoring can satisfy the peculiarities of each target system (Li et al, 2009).

In further virtual hit selection, the priority goes to the ligands which have lower $\Delta \mathrm{G}_{\text {binds }}$ and similar binding modes with the reference (Yang et $a l, 2013)$. A diverse hydrogen bond interaction was demonstrated by all ligands fit into the active site with the corresponding amino acid residues such as GLU323, PRO324, PRO325, LEU345, LEU346, GLU353, TRP393, ARG394 and LYS449. However, among those mentioned amino acid residues, interactions with GLU353 and ARG394 were the most prevalent and it was suggested to be important as these interaction were also possessed by the reference ligand. Therefore, compounds having interaction with these types of residue were proposed to be the virtual hits ready for further optimization. Figure 2 illustrated the superimposition of 27 ligands in the active site of 1G50, whereas Figure 3 illustrated the ligandsprotein interaction of some representative virtual hit compounds.

Table 2. The $\Delta \mathrm{G}_{\text {binding }}$ of 27 ligands were docked into hER $\alpha$ LBD (PDB 1G50).

\begin{tabular}{lcll}
\hline \multicolumn{1}{c}{ Ligands } & $\boldsymbol{\Delta} \mathbf{G}_{\text {bind }}(\mathbf{k c a l} / \mathbf{m o l})$ & \multicolumn{1}{c}{ Amino acid residues } & \multicolumn{1}{c}{ H-bond distance (̊) } \\
\hline Wang3 & -9.2 & ARG394 & 1.98 \\
Wang4 & -8.8 & GLU353, ARG394 & $1.84,1.90$ \\
Latef1 & -7.8 & NHB & NA \\
Latef6 & -7.8 & NHB & NA \\
Latef4 & -7.7 & PRO324, ARG394, LYS449 & $2.44,2.19,1.90$ \\
Latef5 & -7.6 & GLU323, ARG394, LYS449 & $2.27,2.14,2.41$ \\
Wang5 & -7.2 & NHB & NA \\
HWergos58 & -6.8 & TRP393 & 2.12 \\
Marwah6 & -6.8 & NHB & NA \\
Marwah5 & -6.6 & ARG394, LYS449 & $2.07,2.29$ \\
HWergos & -6.5 & NHB & NA \\
Marwah4 & -6.5 & TRP393, ARG394, LYS449 & $2.10,1.69-1.87,2.49$ \\
Wang1 & -6.5 & NHB & NA \\
WP2 & -6.5 & LEU345, LEU346, ARG394 & $1.95,2.49,1.59$ \\
Marwah2 & -6.3 & TRP393, LYS449 & $2.20,1.80$ \\
Wang2 & -6.1 & LYS449 & 2.20 \\
PengLi & -5.9 & PRO325, ARG394 & $2.10,1.86$ \\
Marwah1 & -5.8 & TRP393 & 2.20 \\
Marwah7 & -5.7 & TRP393, LYS449 & $1.93,2.39$ \\
Latef3 & -5.5 & ARG394, LYS449, & $2.02,2.22$ \\
Qin1 & -5 & TRP393 & 1.78 \\
Latef2 & -4.9 & ARG394, LYS449 & $2.42,2.05$ \\
Qin2 & -4.2 & NHB & NA \\
Marwah3 & 0.7 & NHB & NA \\
Qin3 & 3.3 & NHB & NA \\
WP1 & 6.1 & NHB & NA \\
Qin4 & 9.4 & PRO325 & 1.98 \\
\hline NHB = No Hydrogen bond; NA Not Applicable &
\end{tabular}




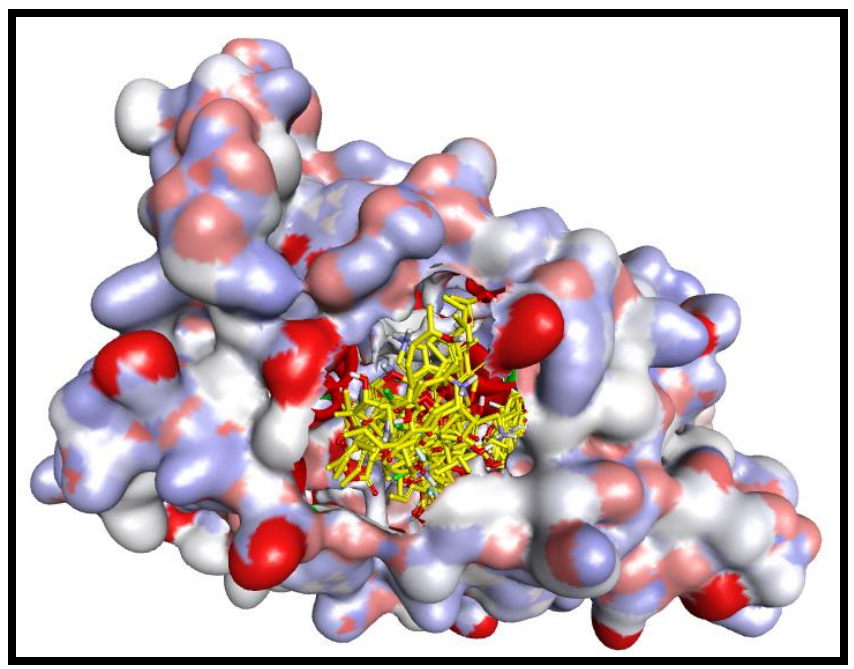

Figure 2. The superimposition of 27 ligands from Chaetomium sp. docked to the active site of HER $\alpha$ (PDB 1G50). The protein was presented as surface and visualized using Discovery Studio 3.5 (www.accelrys.com).

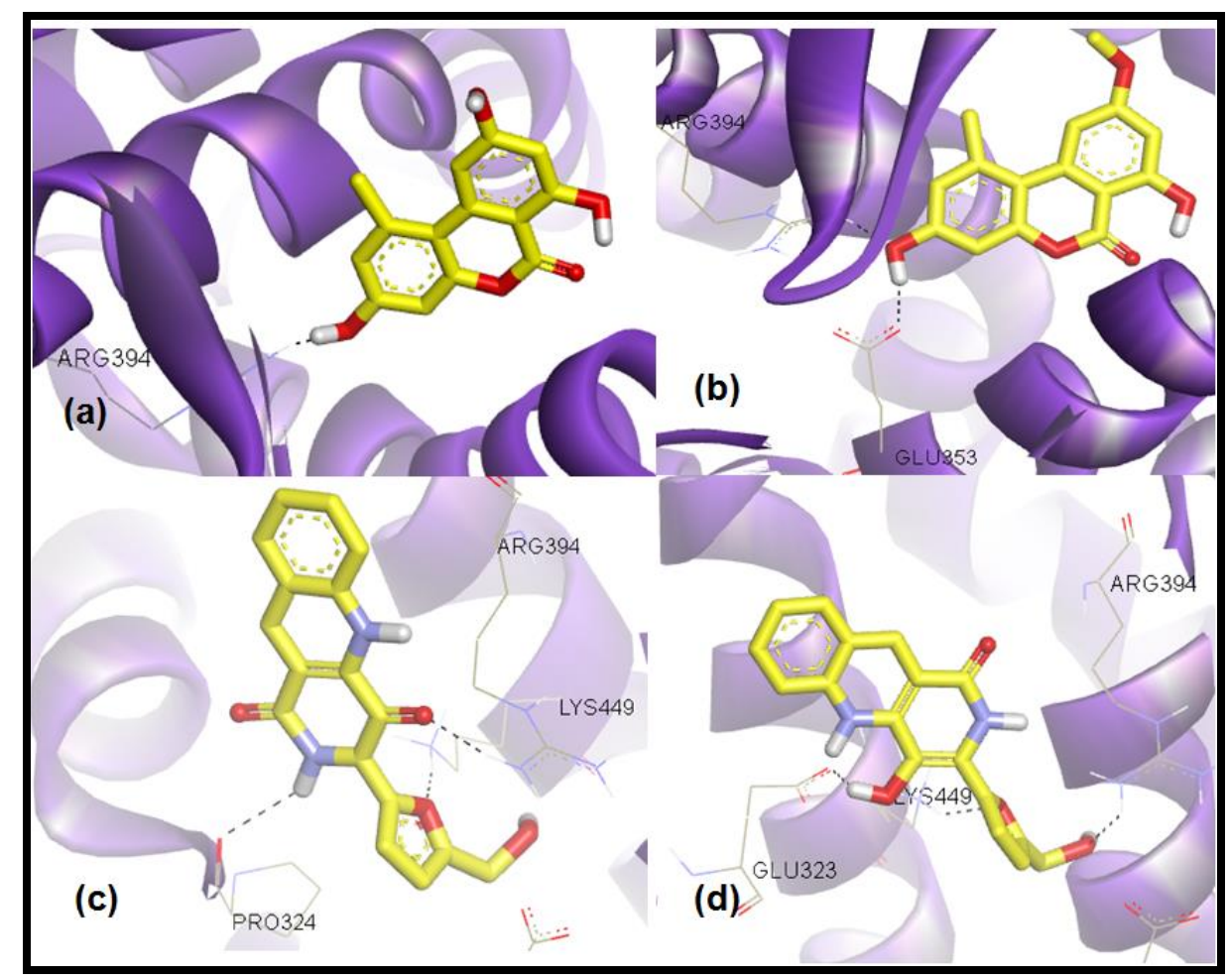

Figure 3. The ligand-binding interaction of four virtual hit compounds from Chaetomium sp. docked to the active site of HER $\alpha$ (PDB 1G50): (a) Wang3 (b) Wang4, (c) Latef4 and (d) Latef5. The protein was presented as ribbon and visualized using Discovery Studio 3.5 (www.accelrys.com).

Compounds bearing xanthone (Wang3 and Wang4) and benzonaphtyridinedione (Latef4 and Latef5) could be good scaffolds for further lead optimization of cytotoxic agent against breast cancer by targeting HER $\alpha$ DNA binding protein domain. Xanthone has been widely published as anticancer due to triple heteroaromatic ring which acts a DNA intercalating agent. The planar system 
of heteroaromatic ring takes over the space in between two layers of nucleic acid pairs and therefore disrupts the shape of the helix. This action occurred at either minor or major groove which prevents the DNA replication as well as its transcription. On the other hand, benzonaphtyridinedione has been reported as tyrosine kinase inhibitors which play roles in signal transduction which regulates the intercellular communication, thus it is a good approach for the therapeutic intervention against pathological processes such as proliferation, inflammation and cancer.

\section{CONCLUSIONS}

Fungal endophyte has been recognized as the new source of bioactive compounds from natural products. One of genus widely cultivate this fungi is Chaetomium sp. spread over plant has been well known in producing high value of compounds for many pharmacological activities. The advantage of this material is its capability to be cultivated in a high yield via biotechnology process compares to the traditional fabrication that needs more plantation. In order to increase the knowledge in developing new anticancer drug from natural product, a molecular docking based virtual screening has been approached to select some hit compounds to be active as breast cancer cytotoxic agent by HER $\alpha$ DNA binding protein domain intervention. Compounds bearing xanthone and benzonaphtyridinedione were selected as the most active ligand against the protein target. In conclusion, Chaetomium sp. is strongly suggested to be developed as the source for bioactive compounds concerning on anticancer agent. However, further retrospective validation is required to have a validated protocol in virtual screening leading to the features of ligand to be suitable as HER $\alpha$ antagonist.

\section{REFERENCES}

Abdel-Lateff, A., 2008. Chaetominedione, a new tyrosine kinase inhibitor isolated from the algicolous marine fungus Chaetomium sp. Tetrahedron Lett, 49(45), 6398-6400.

Eiler, S., et al., 2001. Overexpression, purification, and crystal structure of native ER alpha LBD. Protein Expr Purif, 22(2), 165-73.
Ferlay, J., et al., 2007. Estimates of the cancer incidence and mortality in Europe in 2006. Annals of Oncology, 18(3), 581-592.

Horwitz, K.B. and W. McGuire, 1978. Estrogen control of progesterone receptor in human breast cancer: correlation with nuclear processing of estrogen receptor. Journal of Biological Chemistry, 253(7), 2223-8.

Hussain, M.S., et al., 2012. Current approaches toward production of secondary plant metabolites. Journal of Pharmacy and Bioallied Sciences, 4(1), 10.

Kandavel, D. and S. Sekar, 2015. Endophytic fungi from Phyllanthus amarus Schum. \& Thonn. capable of producing phyllanthin, hypophyllanthin and/or related compounds. Int J Pharm Pharm Sci, 7(5).

Kitchen, D.B., et al., 2004. Docking and scoring in virtual screening for drug discovery: methods and applications. Nature reviews Drug discovery, 3(11), 935-949.

Kumar, A., et al., 2013. Isolation, purification and characterization of vinblastine and vincristine from endophytic fungus Fusarium oxysporum isolated from Catharanthus roseus. PloS one, 8(9), e71805.

Lane, N.E., 2006. Epidemiology, etiology, and diagnosis of osteoporosis. American Journal of Obstetrics and Gynecology, 194(2), S3S11.

Li, H., et al., 2009. An effective docking strategy for virtual screening based on multiobjective optimization algorithm. BMC Bioinformatics, 10(1), 58.

Li, H.-Q., et al., 2011. Antifungal metabolites from Chaetomium globosum, an endophytic fungus in Ginkgo biloba. Biochemical Systematics and Ecology, 39(4-6), 876-879.

Li, P., et al., 2015. Paeonol produced by Chaetomium sp., an endophytic fungus isolated from Paeonia suffruticosa. Phytochem Lett, 13, 334-342.

Marwah, R.G., et al., 2007. Musanahol: a new aureonitol-related metabolite from a Chaetomium sp. Tetrahedron, 63(34), 81748180.

Patrick, G.L., 2013. An Introduction to Medicinal Chemistry. Fifth Edition. Oxford University Press. 
Qin, J.-C., et al., 2009. Bioactive metabolites produced by Chaetomium globosum, an endophytic fungus isolated from Ginkgo biloba. Bioorg Med Chem Lett, 19(6), 15721574.

Ruch, R.J., Cheng, S.-J. and J.E. Klaunig, 1989. Prevention of cytotoxicity and inhibition of intercellular communication by antioxidant catechins isolated from Chinese green tea. Carcinogenesis, 10(6), 1003-1008.

Stierle, A., G. Strobel, and D. Stierle, 1993. Taxol and taxane production by Taxomyces andreanae, an endophytic fungus of Pacific yew. Science, 260(5105), 214-216.

Strobel, G.A., 2003. Endophytes as sources of bioactive products. Microb Infect, 5(6), 535544.

Wang, Y., et al., 2012. Bioactive metabolites from Chaetomium globosum L18, an endophytic fungus in the medicinal plant Curcuma wenyujin. Phytomedicine, 19(3), 364-368.

Wang, M.-H., et al., 2015. Stereochemical determination of tetrahydropyran-substituted xanthones from fungus Chaetomium murorum. Chin Chem Lett, 26(12), 15071510.

Yang, S.-X., et al., 2011. Toxic polyketides produced by Fusarium sp., an endophytic fungus isolated from Melia azedarach. Bioorganic \& Medicinal Chemistry Letters, 21(6), 1887-1889.

Yang, L., et al., 2013. How much do van der Waals dispersion forces contribute to molecular recognition in solution? Nat Chem, 5(12), 1006-1010.

Zhang, Q., et al., 2012. Chemical and bioactive diversities of the genus Chaetomium secondary metabolites. Mini reviews in medicinal chemistry, 12(2), 127-148.

Zhao, J., et al., 2010. Endophytic fungi for producing bioactive compounds originally from their host plants. Curr Res, Technol Educ Trop Appl Microbiol Microbial Biotechnol, 1, 567-576. 